\title{
Development of a robust DNA quality and quantity assessment qPCR assay for targeted next-generation sequencing library preparation
}

\author{
JENNIFER DANG ${ }^{1}$, PEDRO MENDEZ ${ }^{2}$, SHARON LEE ${ }^{1}$, JAMES W. KIM $^{1}$, JUN-HEE YOON ${ }^{2}$, \\ THOMAS W. KIM ${ }^{1}$, CHARLES J. SAILEY ${ }^{1,4,5}$, DAVID M. JABLONS ${ }^{2,3}$ and IL-JIN KIM ${ }^{2,3}$ \\ ${ }^{1}$ CureSeq Inc., Brisbane, CA 94005; ${ }^{2}$ Thoracic Oncology Laboratory, Department of Surgery, \\ ${ }^{3}$ Comprehensive Cancer Center, University of California San Francisco, San Francisco, CA 94115; \\ ${ }^{4}$ Purity Laboratories, Lake Oswego, OR 97035; ${ }^{5}$ Molecular Testing Labs, Vancouver, WA 98684, USA
}

Received June 7, 2016; Accepted August 1, 2016

DOI: 10.3892/ijo.2016.3654

\begin{abstract}
Next-generation sequencing (NGS) is becoming a standard for genetic analyses of clinical samples. DNAs retrieved from formalin-fixed, paraffin-embedded (FFPE) tissue specimens are commonly degraded, and specimens such as core biopsies are sometimes too small to obtain enough DNA for NGS applications. Thus, it is important to measure both the DNA quantity and quality accurately from clinical samples. However, there is no standard method for DNA quantity and quality analyses for NGS library preparation. We tested four different methods (PicoGreen, Qubit ${ }^{\circledR}$ fluorometry, TaqMan and SYBR-Green-based qPCR assay) and compared each to RNase $\mathrm{P}$ TaqMan as a reference control. We found that SYBR-Green-based qPCR assay provides a consistent and accurate DNA quantification while keeping its cost relatively low and the throughput high. We designed a dual-probe SYBR-Green qPCR assay for DNA quantity and quality assessment for targeted NGS library preparation. This assay provides a Dscore (degradation score) of the interrogated DNA by analyzing two different sizes of amplicons. We show an example of a clinical sample with a very high Dscore (high degradation). With a regular DNA quantification, without considering the degradation status, no correct NGS libraries were obtained. However, after optimizing the library condition by considering its poor DNA quality, a reasonably good library and sequencing results were obtained. In summary, we developed and presented a new DNA quantity and quality analysis qPCR assay for the targeted NGS library preparation.
\end{abstract}

Correspondence to: Dr Il-Jin Kim or Dr David M. Jablons, Thoracic Oncology Laboratory, Department of Surgery, University of California San Francisco, 2340 Sutter Street, Room N219, San Francisco, CA 94115, USA

E-mail: Il-Jin.kim@ucsf.edu

E-mail: david.jablons@ucsf.edu

Key words: next-generation sequencing, DNA quality and quantity, qPCR, dual probes
This assay may be mostly efficient for the clinical samples with high degradation and poor DNA quality.

\section{Introduction}

The development of the next-generation sequencing (NGS) allows us to do many different types of genetic and genomic analyses in a high throughput way (1-4). Completion of the whole coding exon (exome) or genome sequencing takes several months to years using technologies such as Sanger sequencing. But it takes only several days or weeks using NGS, providing a tremendous amount of genomic information which can be used for developing therapeutic targets or diagnostic markers (1-5). Another significant trend toward NGS is the application of targeted sequencing for selected genes related to clinical or biological significance such as cancer driver genes or the drug target genes such as EGFR and BRAF (6-9). After completing large scale collaborative sequencing projects such as The Cancer Genome Atlas (TCGA) and identifying many cancer driving mutations (10-14), targeted sequencing approaches designed only for biologically or clinically proven genes are becoming both more popular and a standard for precision medicine approaches in hospitals and clinics (6-9).

There are several ways to enrich for selecting genes or coding regions $(5,6,15-17)$. The most frequently used targeted enrichment methods are amplification by PCR $(6,7)$ or hybrid capture (15-17). Hybrid capture method tends to require more DNA (i.e. 50-100 ng) than an amplification method (i.e. $10 \mathrm{ng}$ ) (6,7,15-17). Each method requires different amount of DNA and suggests a different quantity and quality check of DNA for their library preparation. However, there is no gold standard for DNA quality and quantity assessment in targeted NGS. The lack of standard assessment may cause inconsistent and non-reproducible results among different targeted enrichment methods and different NGS platforms (6,7,15-17).

A lot of clinical samples from hospitals are incorrectly processed, or stored, and may be degraded or too small to extract enough high quality DNA. Formalin-fixed, paraffinembedded (FFPE) tissues are the most frequently used sample type for diagnosis and genetic screening in hospitals. DNAs 
extracted from FFPE tissues are well known to be degraded and fragmented, thus, special caution is needed for molecular genetic analyses of DNA derived from FFPE tissues (7). A biopsy specimen, another frequently used sample type in hospitals, is also challenging for extracting enough high quality DNA due to its small sample (cancer) size. A small amount of DNA may be amplified using a whole genome amplification (WGA) method for the downstream genetic analyses. But it is known that amplification biases affect results and cause false positive and negative results $(18,19)$. Thus, it is very important to measure correctly and efficiently the quality and quantity of DNA extracted from challenging clinical samples for genetic analyses such as NGS.

Several different DNA quantity and quality measurement methods have been used for NGS and other genetic analyses. UV spectroscopy or spectrophotometry (i.e. NanoDrop) may be simplest and easiest way to measure DNA or other materials in certain ranges of wavelength (20). However, UV spectrophotometers detect not only DNA, but also UV-absorbing materials like RNA, protein and phenol (20) and are not sensitive enough to detect a small amount of DNA. Another DNA quantitation method is the detection of double-stranded DNA (dsDNA) using a fluorescent dye (21). PicoGreen is a fluorescent dye that preferentially binds to dsDNA (22). Thus, a PicoGreen-based dsDNA measurement can provide more specific results than

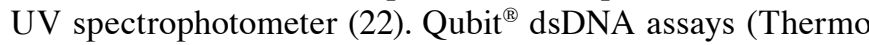
Fisher Scientific) also detect dsDNA using a fluorescent dye and fluorometer (23). Another commonly used method is a capillary electrophoresis of DNA or RNA in a chip (24). Agilent Bioanalyzer may be the best example of this capillary electrophoresis method (25). Bioanalyzer provides quantity, size and quality of measured DNA and RNA and may be a gold standard method especially for RNA quantity and quality analyses. However, this method requires several experimental steps and is relatively more complex than other methods. The cost of Bioanalyzer analysis may also be the highest among others. The last method is a qPCR-based DNA quantification $(21,23,26,27)$. qPCR is widely used for gene expression, mutation and various genotyping analyses. TaqMan (Thermo Fisher Scientific) may be the most common qPCR-based assay using unlabeled PCR primers and fluorescent labeled probe $(21,23,26,27)$. Another commonly used method is a SYBRGreen-based qPCR assay. Like PicoGreen, SYBR-Green also preferentially binds dsDNA and has widely been used for various qPCR assay $(21,23,26,27)$. Unlike TaqMan assays, no specific fluorescent probe is required thus, SYBR-Green is cheaper and provides more flexibility for assay design.

In the present study, we tested four commonly used DNA measurement methods for their accuracy, cost and user-friendliness to finally select the best method of DNA quality and quantity assessment for NGS library preparation. As there is no gold standard for DNA analyses for NGS, and each NGS method suggests a different method, we believe that this comprehensive analyses of four different methods will help researchers find the best and most efficient method for targeted NGS.

\section{Materials and methods}

Experimental design. To evaluate quantitation techniques and determine the optimal method for quantitation of DNA derived from FFPE tissue samples for NGS library preparation, we compared four alternative methods: i) fluorometry using PicoGreen; ii) Qubit ${ }^{\circledR}$ fluorometer (Thermo Fisher Scientific); iii) an in-house designed duplexed TaqMan qPCR assay; and iv) an in-house dual-probe SYBR-Green qPCR assay to qPCR TaqMan RNase P. After determining which of the methods quantified samples consistently when compared to RNase P, we then compared NGS library performance based on yield for samples prepped with inputs based on RNase P and the similar alternative quantitation method.

In-house qPCR designs. Because the chronic exposure to UV light, skin melanoma is among the top three tumor types with high mutation rate (28). First, we interrogated the genomic mutational landscape from 250 skin melanoma cases (TCGA; http://www.cbioportal.org/) that had available data from exome sequencing and gene copy number analysis to identify those genes with the lowest rate of genetic alterations $(<1 \%)$. Second, the list of candidate genes was filtered by using the exome and gene copy number data from more than 17,000 tumors (TCGA) to identify genes or loci with less genetic alterations ( $<4 \%$ in gene copy number, and never homozygous deletions and $<1.5 \%$ of SNV) across the 50 most frequent tumor types. Third, the genes that had annotated pseudogenes (29) were discarded for design, as pseudogenes can cause an over quantification of DNA due to the extra copies in the genome. Fourth, the genes located in sexual chromosomes were excluded. Fifth, to ensure a DNA specific quantification, the oligonucleotides were designed in the intronic or intergenic regions of the targeted genes. Sixth, the specificity of the oligonucleotides was verified by primer blast (NCBI) against the Human genome reference sequence. Finally, the lack of polymorphic variants in the primer binding sites was tested and confirmed by SNPCheck V3.0 (https://secure.ngrl.org.uk/SNPCheck/ snpcheck.htm). With these selection criteria, we selected three loci, 22q12.3, 14q24.1 and 15q24.3, with minimal copy number alterations across different types of cancer samples.

We set out to create a two-assay qPCR quantitation method with different amplicon sizes, to allow the assessment of the relative quality of DNA by comparing the concentrations of the smaller amplicon(s) to that of the larger amplicon(s). Thus, six sets of primers for small amplicons ( $\leq 96 \mathrm{bp})$, three sets of large amplicons (<190 bp) and corresponding TaqMan probes were designed in conserved regions of the Human Genome as alternative quantitation assays to TaqMan RNase P. The short amplicons are located at chromosome 22q12.3 and 14q24.1, while the longest amplicons are located at 22q12.3 and 15q24.3. Human genomic DNA (Bioline, Taunton, MA, USA), was serially diluted to construct a standard curve ranging from 40 to $0.064 \mathrm{ng} / \mu \mathrm{l}$ and run on a QuantStudio 6 (Thermo Fisher Scientific) in accordance with the manufacturer's instructions to determine the efficiency of the primer sets with SYBR-Green chemistry using SYBR ${ }^{\circledR}$ GreenER $^{\mathrm{TM}}$ Dye (Thermo Fisher Scientific), a modified SYBR-Green I dye. Inefficient primer pairs, or primer pairs showing more than a single product in their melt curves were excluded from further testing. Efficient primer pairs were then run with their corresponding TaqMan probe and Human genomic DNA (Bioline) was serially diluted to construct a standard curve ranging from 100 to $0.16 \mathrm{ng} / \mu \mathrm{l}$ and run to determine the efficiency of the 

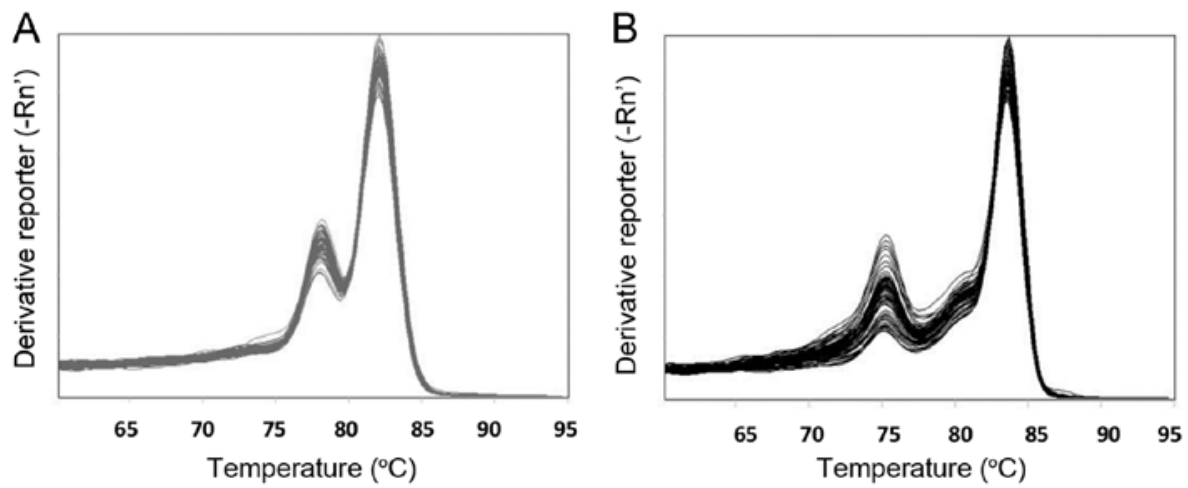

Figure 1. Melting curves of qPCR reactions from dual-probe SYBR-Green assay. Representative melting curves for (A) DS1 and (B) DS2 amplicons from the dual-probe SYBR-Green assay.

primers/probe with TaqMan chemistry. One of each efficient small and each efficient large amplicon was then combined to make 10 duplex TaqMan assays and tested for efficiency with a standard curve ranging from 40 to $0.8 \mathrm{ng} / \mu$ l. One duplex was chosen as the final in-house design for its consistent efficiency and deemed QC1 (small amplicon) and QC2 (large amplicon). Similarly, the small amplicon designs were paired creating pools of two sets of small amplicon oligos, and large amplicon designs were also paired creating pools of two large amplicon oligos. Small amplicon pairs and large amplicon combinations were then tested for efficiency with a standard curve ranging from 40 to $0.8 \mathrm{ng} / \mu \mathrm{l}$. One of the small amplicon combinations and one of the large amplicon combinations was chosen as the final in-house design for their melt curve profiles (Fig. 1) and consistent efficiencies to form a dual-probe SYBR-Green assay that interrogates multiple regions of the genome. The combinations were deemed DS1 (small amplicon combination) and DS2 (large amplicon combination).

Clinical specimens. DNA samples were used for comparison and evaluation quantitation methods including 26 frozen and 45 FPPE cancer tissue samples collected under a protocol (\#11-06107) approved by the Committee for Human Research at the University of California, San Francisco and one cancer FFPE cancer cell line, A549. Samples P12 and P37 were collected after prior consent and patient approval. The sequencing data of sample P12 and P37 were collected from the original clinical run in a certified Clinical Laboratory Improvement Amendments (CLIA) laboratory (Purity Laboratories, Lake Oswego, OR, USA) and have been fully de-identified.

FFPE tissue processing and DNA extraction. Each FFPE tissue section, ranging in size from 5 to $10 \mu \mathrm{m}$ in thickness with no more than $2.25 \mathrm{~cm}^{2}$ of tissue area, was deparaffinized by submersion in xylene for $10 \mathrm{~min}$ at room temperature and air dried for at least $10 \mathrm{~min}$. DNA extraction was performed on the deparaffinized tissue section with the UltraRapid FFPE DNA Extraction kit (CureSeq, Inc., Brisbane, CA, USA) in accordance with the manufacturer's instructions and as previously described (7). Five of the 45 samples were also extracted with the AllPrep DNA/RNA FFPE kit (Qiagen) in accordance with the manufacturer's instructions for extraction method comparison. For the UltraRapid FFPE DNA extraction, $5 \mu 1$ of Solution A (CureSeq) was spread over the FFPE tissue sections to hydrate the tissue before it was scraped and transferred to a PCR tube containing $70 \mu \mathrm{l}$ of Solution A. The tube containing tissue was incubated for $5 \mathrm{~min}$ at $99^{\circ} \mathrm{C}$, before the addition of $10 \mu \mathrm{l}$ of Solution B (CureSeq). After the addition of Solution B, samples were mixed by shaking, briefly spun down, and then incubated for $5 \mathrm{~min}$ at $60^{\circ} \mathrm{C}$ followed by $5 \mathrm{~min}$ at $99^{\circ} \mathrm{C}$. Samples were then centrifuged at $1,000 \mathrm{x}$ g for $1 \mathrm{~min}$ at room temperature, and the supernatant containing DNA was collected and transferred to a new tube for quantification. For the AllPrep DNA/RNA FFPE extraction, $7 \mu$ of buffer PKD (Qiagen) was spread over the FFPE tissue sections to hydrate the tissue before it was scraped and transferred to a $1.5 \mathrm{ml}$ microcentrifuge tube containing $150 \mu 1$ of buffer PKD. After the addition of proteinase $\mathrm{K}$, tissue was partially digested at $56^{\circ} \mathrm{C}$ for $15 \mathrm{~min}$, then spun down for $15 \mathrm{~min}$ at $20,000 \mathrm{x} \mathrm{g}$. The supernatant was removed, and the pellet was suspended with buffer ATL (Qiagen). Additional proteinase $\mathrm{K}$ was added and the re-suspended pellet was incubated at $56^{\circ} \mathrm{C}$ for $1 \mathrm{~h}$, followed by $90^{\circ} \mathrm{C}$ for $2 \mathrm{~h}$. After incubation, buffer AL (Qiagen) and ethanol was added before samples were transferred to a QIAamp MinElute spin column (Qiagen) for column purification. Purified DNA was eluted in $85 \mu \mathrm{l}$ of nuclease-free water for quantification.

Frozen tissue processing and DNA extraction. DNA from frozen tissues was extracted with DNeasy Blood \& Tissue kit (Qiagen) following the manufacturer's instructions. Briefly, up to $25 \mathrm{mg}$ of tissue was disrupted by using TissueLyser LT for $5 \mathrm{~min}$ at $50 \mathrm{~Hz}$. The tissue samples were digested with ATL buffer in presence of proteinase $\mathrm{K}$ at $56^{\circ} \mathrm{C}$ for at least $1 \mathrm{~h}$. The DNA was purified by using QIAmp MinElute columns, and eluted in $200 \mu l$ of AE buffer.

PicoGreen assay. The stock DNA and the corresponding 1:5 diluted DNA derived from FFPE tissue was quantified by using Quant-iT ${ }^{\mathrm{TM}}$ PicoGreen dsDNA Assay kit (Thermo Fisher Scientific) as follows: $2 \mu \mathrm{l}$ of sample DNA were diluted 1:5 vol/vol in 1x TE, containing 1:100 diluted PicoGreen reagent in a final reaction volume of $40 \mu \mathrm{l}$ per reaction. Human genomic DNA (Bioline) was serially diluted to construct a standard curve ranging from 2.5 to $0.08 \mathrm{ng} / \mu \mathrm{l}$ of DNA. Each 
experimental sample or dilution point of the standard curve was assayed in triplicates. The fluorometric quantitation was performed in the Synergy HTX platform (BioTek Instruments, Inc., Winooski, VT, USA).

Qubit assay. The Qubit dsDNA HS (high sensitivity) assay kit (Thermo Fisher Scientific) was used to quantify DNA derived from FFPE tissue in accordance with the manufacturer's instruction. Samples were prepared as follows: $2-5 \mu 1$ of stock DNA or 1:5 diluted (vol/vol) DNA was added to 195-198 $\mu \mathrm{l}$ of the Qubit ${ }^{\circledR}$ working solution for final volume of $200 \mu 1$. The $200 \mu \mathrm{l}$ DNA and working solution were then incubated at room temperature for at least 2 min before quantitation was performed with the Qubit 3.0 fluorometer (Thermo Fisher Scientific).

TaqMan qPCR assay. DNA derived from FFPE tissue was quantified by the TaqMan duplex assay and run on a QuantStudio 6 qPCR platform (Thermo Fisher Scientific), following the manufacturer's instructions. The TaqMan duplex assay is a fluorescent-based qPCR assay consisting of two sets of primer probe oligos sets, QC1 and QC2. QC1 (amplicon length $\leq 96 \mathrm{bp}$ ) measures short DNA fragments for quantitation and QC2 measures the presence long DNA fragments (amplicon length $<190 \mathrm{bp}$ ). Human genomic DNA (Bioline) was serially diluted to construct a standard curve ranging from 40 to $0.8 \mathrm{ng} / \mu \mathrm{l}$. Each experimental DNA sample or dilution point of the standard curve was run in triplicate for both QC1 and QC2 assays. The qPCR reagent reaction volumes for both QC1 and QC2 were as follows: $2 \mu$ of stock or diluted DNA was mixed with $3.75 \mu \mathrm{l}$ of $2 \mathrm{X}$ PCR Master Mix, $0.375 \mu \mathrm{l}$ of 20X QC1 or QC2 assay and $1.375 \mu \mathrm{l}$ of water. The cycling conditions for the qPCR reaction was as follows: $10 \mathrm{~min}$ at $50^{\circ} \mathrm{C}, 2 \mathrm{~min}$ at $95^{\circ} \mathrm{C}, 40$ cycles of $15 \mathrm{sec}$ at $95^{\circ} \mathrm{C}$ and $1 \mathrm{~min}$ at $60^{\circ} \mathrm{C}$. ROX was used as passive reference.

Dual-probe SYBR-Green qPCR assay. DNA derived from FFPE and frozen tissue was quantified by the two primer pair SYBR-Green qPCR assay on a QuantStudio 6 qPCR platform (Thermo Fisher Scientific), following the manufacturer's instructions. The two primer pair SYBR-Green system is a fluorescent-based qPCR assay consisting of two sets of oligos, DS1 and DS2. DS1 (amplicons length $\leq 85 \mathrm{bp}$ ) measures short DNA fragments for quantitation and DS2 measures the presence of long amplicons (length $<190 \mathrm{bp}$ ). Human genomic DNA (Bioline) was serially diluted to construct a standard curve ranging from 40 to $0.8 \mathrm{ng} / \mu 1$. Each experimental DNA sample or dilution point of the standard curve was run in triplicate for both DS1 and DS2 assays. The qPCR reagent reaction volumes for both DS1 and DS2 were as follows: $2 \mu \mathrm{l}$ of stock or diluted DNA was mixed with $10 \mu 1$ of 2x PCR Master Mix, $1 \mu 1$ of 20X DS1 or DS2 assay oligonucleotides and $7 \mu \mathrm{l}$ of water. The cycling conditions for the qPCR reaction was as follows: $2 \mathrm{~min}$ at $50^{\circ} \mathrm{C}, 2 \mathrm{~min}$ at $95^{\circ} \mathrm{C}, 40 \mathrm{qPCR}$ cycles of $15 \mathrm{sec}$ at $95^{\circ} \mathrm{C}$ and $1 \mathrm{~min}$ at $60^{\circ} \mathrm{C}, 15 \mathrm{sec}$ at $95^{\circ} \mathrm{C}, 1 \mathrm{~min}$ at $60^{\circ} \mathrm{C}$, and $15 \mathrm{sec}$ at $95^{\circ} \mathrm{C}$. ROX was used as passive reference.

TaqMan RNase P qPCR. DNA derived from FFPE tissue was quantified by TaqMan ${ }^{\circledR}$ Copy Number Reference Assay, human, RNase P (Thermo Fisher Scientific) and run on a
QuantStudio 6 qPCR platform (Thermo Fisher Scientific), following the manufacturer's instructions. Human genomic DNA (Bioline) was serially diluted to construct a standard curve ranging from 40 to $0.8 \mathrm{ng} / \mu \mathrm{l}$. Each experimental DNA sample or dilution point of the standard curve was run in triplicate. The qPCR reagent reaction volumes for RNase P reactions were as follows: $2 \mu \mathrm{l}$ of stock and or diluted DNA was mixed with $3.75 \mu \mathrm{l}$ of $2 \mathrm{X}$ PCR Master Mix, $0.375 \mu \mathrm{l}$ of 20X RNase P assay oligonucleotides and $1.375 \mu \mathrm{l}$ of water. The cycling conditions for the qPCR reaction was as follows: $10 \mathrm{~min}$ at $50^{\circ} \mathrm{C}, 2 \mathrm{~min}$ at $95^{\circ} \mathrm{C}, 40$ cycles of $15 \mathrm{sec}$ at $95^{\circ} \mathrm{C}$ and $1 \mathrm{~min}$ at $60^{\circ} \mathrm{C}$. ROX was used as passive reference.

Targeted NGS library preparation. The targeted cancer NGS panel (NextDay Seq-Pan Cancer HotSpot Panel kit; CureSeq) was used for library preparation (7). The libraries were prepared using a $10 \mathrm{ng}$ input of DNA based on quantitation by TaqMan RNase P, the TaqMan QC2, SYBR-Green DS1 and SYBR-Green DS2. DNA was added to a multiplexed PCR reaction and run for 22 cycles. The PCR products were then ligated to universal adapters and barcodes. The ligated PCR products were purified by using a magnetic bead-based protocol and eluted in $30 \mu \mathrm{l}$ of $1 \mathrm{X}$ LTE buffer to produce the final purified libraries. Each library $(1 \mu \mathrm{l})$ was run on a High Sensitivity DNA chip (Agilent Technologies, Santa Clara, CA, USA; cat. no. 5067-4626) to evaluate the quality and library yield. The yield from each library (pmol/l) was determined by a smear analysis of the electropherogram in the 245-400 bp range, using the Bioanalyzer 2100 platform (Agilent Technologies) and software.

Library yield comparisons. Libraries were generated based on QC2, DS1 and DS2 quantitation to compare to libraries generated by RNase P quantitation. QC1 was not chosen for the library preparation comparison due to its similar quantitation values to RNase P. For the QC2 and RNase P comparison 17 samples were chosen based on their Dscore (ratio of the concentration of QC1 divided by the concentration of QC2) for NDS library prep and divided into 3 categories: i) 'High integrity DNA' with Dscores ranging from 0.95 to 1.50 which were expected to generate libraries with similar or better yields than libraries constructed based on the corresponding RNase P quantitation; ii) 'Rescue samples' with high concentrations by RNase $\mathrm{P}(>10 \mathrm{ng} / \mu \mathrm{l})$ and low concentrations of QC2 $(<10 \mathrm{ng} / \mu \mathrm{l})$ which were suspected to perform poorly when using RNase $\mathrm{P}$ concentrations and perform better with QC2 quantitation; and iii) 'Poor integrity DNA' with high Dscores $(>4)$ or no expression for QC2 which were expected to perform poorly with both quantitation methods, but show greater yields for QC2 based libraries compared to libraries based on than RNase P. For the DS1, DS2 and RNase P comparison 11 samples were initially chosen based on their range of Dscore (the ratio of the concentration of DS1 divided by the concentration of DS2). After the initial 11 samples, 25 additional samples were prepared based on DS1 and RNase P for comparison.

\section{Results}

Comparison of DNA quantitation by PicoGreen vs. TaqMan RNase P. PicoGreen on average, quantified DNA samples 


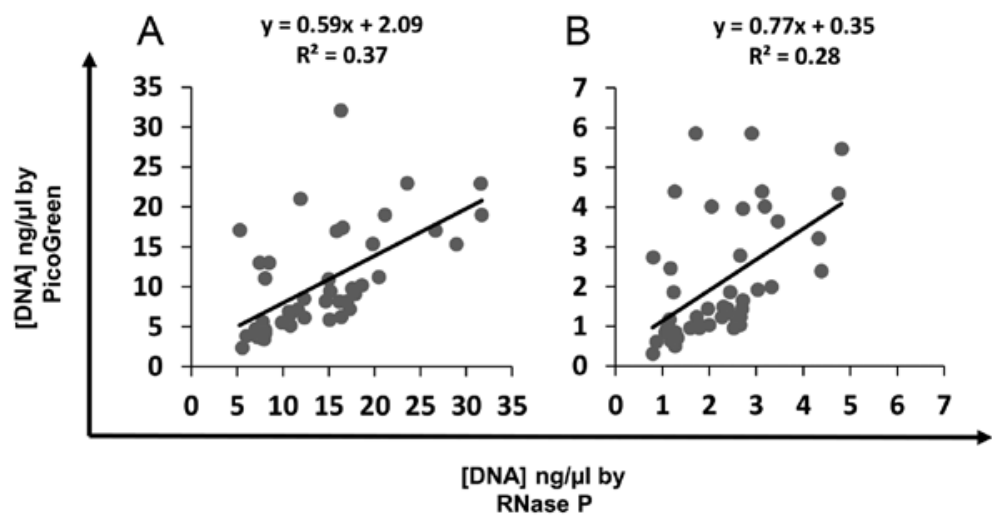

Figure 2. Correlation of FFPE sample concentrations by PicoGreen quantitation and TaqMan RNase P quantitation. (A) Regression analysis of 46 Stock DNA sample concentrations measured by PicoGreen and TaqMan RNase P. (B) Regression analysis of 46 1:5 diluted DNA sample concentration measured by PicoGreen and TaqMan RNase P.

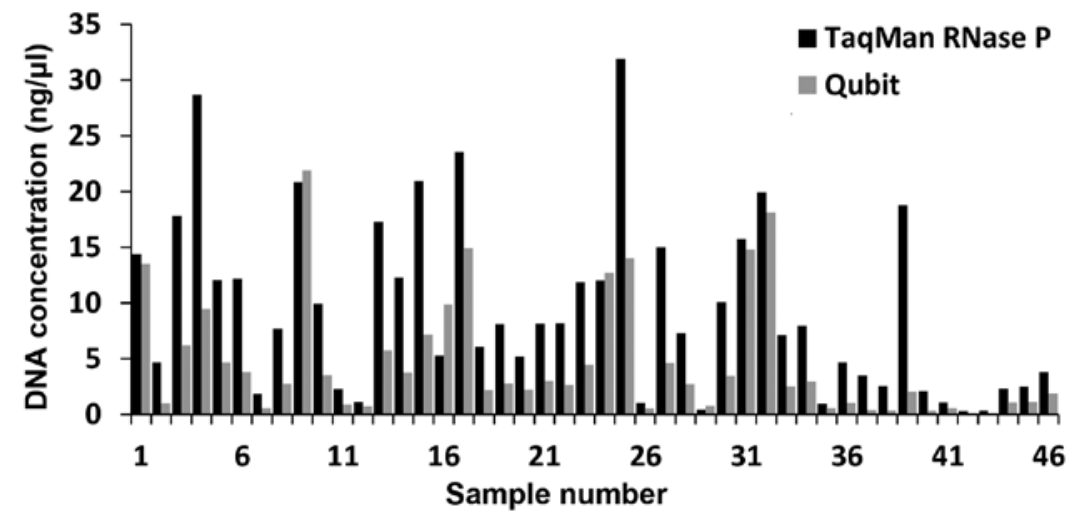

Figure 3. Concentration of FFPE samples by TaqMan RNase P quantitation and Qubit ${ }^{\circledR}$ quantitation. Concentration of 46 FFPE samples quantified by TaqMan RNase P (black) and Qubit ${ }^{\circledR}$ (gray).

at lower concentrations compared to TaqMan RNase P quantitation. There was a weak correlation between DNA concentrations by PicoGreen and RNase P, for stock DNA (Fig. 2A; slope $=0.59 ; \mathrm{R}^{2}=0.37$ ) and 1:5 diluted DNA samples (Fig. 2B; slope $=0.77 ; \mathrm{R}^{2}=0.28$ ). The DNA concentration measured by RNase $\mathrm{P}$ was on average 1.41 - and 1.58 -fold higher than measured by fluorometry for both the 1:5 dilution and stock DNA FFPE tissue specimens, respectively.

Comparison of DNA quantitation by Qubit vs. TaqMan RNase $P$. In agreement with the data observed in the PicoGreen comparison against TaqMan RNase P assay, the DNA was consistently under quantified by Qubit fluorometry compared to RNase P. On average the DNA concentration measured by RNase $\mathrm{P}$ was 2.88 times greater than the concentration measured by Qubit, but the fold change ranged from 0.53 to 9.24 times greater (Fig. 3).

Comparison of DNA quantitation by in-house duplex TaqMan vs. TaqMan RNase P. The DNA concentration measured by QC1 (TaqMan small amplicon) and by TaqMan RNase P, showed a very strong correlation (slope $=1.08 ; \mathrm{R}^{2}=0.86$; Fig. 4A), while the data obtained by QC2 (large amplicon) showed weak correlation, with an important deviation in the slope, in the TaqMan RNase P-values (slope $=0.70 ; \mathrm{R}^{2}=0.36$;
Fig. 4B). QC1 consistently quantified DNA at slightly higher concentrations than TaqMan RNase P, while QC2 under quantified when compared to RNase P. On average the DNA concentration measured by RNase $\mathrm{P}$ was 0.73 less than QC1 and 1.62 times greater than QC2.

Comparison of DNA quantitation by in-house duplex SYBRGreen qPCR vs. TaqMan RNase P. The DNA concentration by DS1 (small amplicon) was on average 1.13-fold higher than by TaqMan RNase $\mathrm{P}$ although their correlation proved to be very strong (slope $=0.82 ; \mathrm{R}^{2}=0.69 ;$ Fig. $4 \mathrm{C}$ ). On other hand, RNase $\mathrm{P}$ measurements were on average 1.70 times greater than the concentration measured by DS2, with moderate correlation (slope $=0.61 ; \mathrm{R}^{2}=0.55 ;$ Fig. 4D).

Comparison of DNA quantitation by in-house duplex TaqMan vs. in-house duplex SYBR-Green qPCR. The DNA concentration measured by TaqMan QC1 and by SYBR DS1, showed a very strong correlation (slope $=1.06 ; \mathrm{R}^{2}=0.82 ;$ Fig. 4E). TaqMan QC1 quantified DNA at higher concentrations than SYBR DS1. On average the DNA concentration measured by QC1 was 1.29 times greater than DS1. Likewise, the DNA concentration measured by TaqMan QC2 and by SYBR DS2, showed a very strong correlation (slope $=1.35 ; \mathrm{R}^{2}=0.90 ;$ Fig. $4 \mathrm{~F}$ ) and TaqMan QC2 quantified DNA at slightly higher concentrations than 

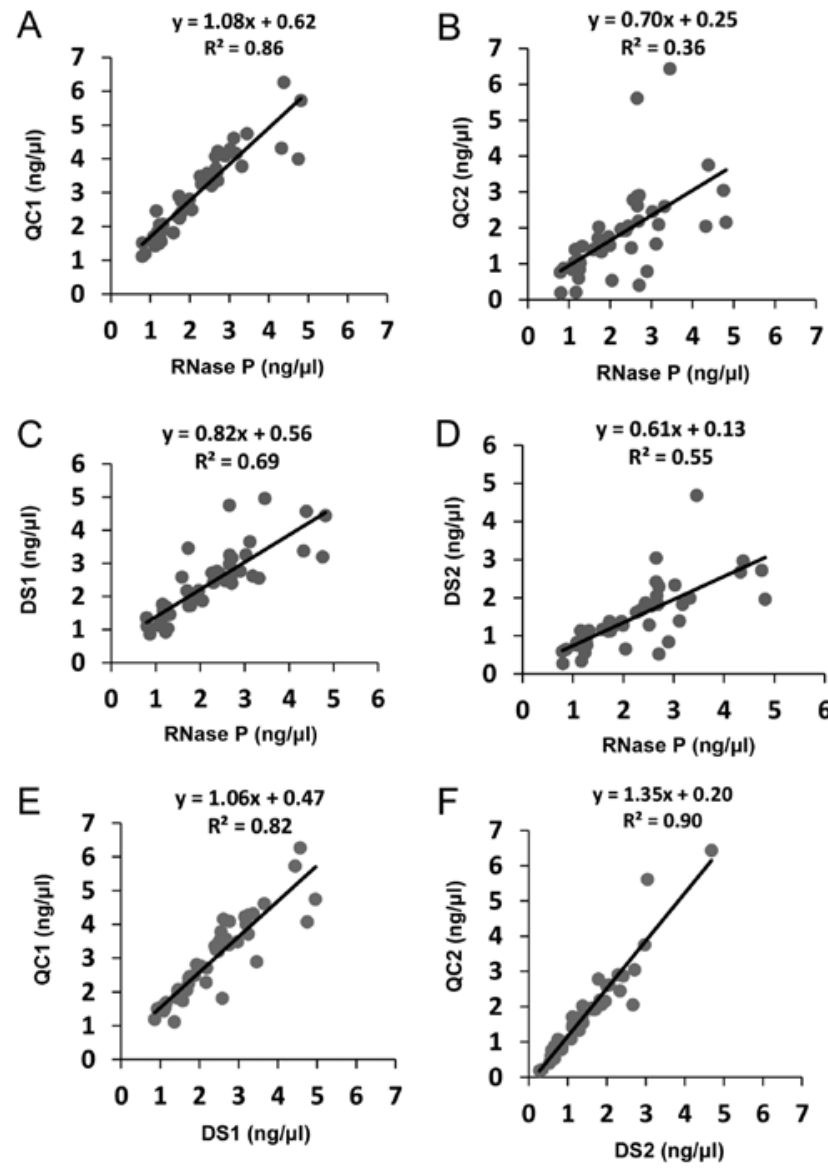

Figure 4. Correlation of FFPE sample concentrations by QC1, QC2, DS1 and DS2 quantitation with TaqMan RNase P quantitation. (A) Regression analysis of 46 diluted DNA samples measured by $\mathrm{QC1}$ and TaqMan RNase $\mathrm{P}$, (B) by QC2 and TaqMan RNase P, (C) by DS1 and TaqMan RNase P, (D) by DS2 and TaqMan RNase P, (E) by QC1 and DS1 and (F) by QC2 and DS2.

Table I. Library yields for libraries prepped with input based on TaqMan RNase P and TaqMan QC2.

\begin{tabular}{lrrr}
\hline & & \multicolumn{2}{c}{$\begin{array}{c}\text { Library yield by input } \\
\text { method (pM) }\end{array}$} \\
\cline { 3 - 4 } Sample category & Sample no. & RNase P & \multicolumn{1}{c}{ QC2 } \\
\hline High integrity DNA & 1 & $11,340.00$ & $12,450.20$ \\
High integrity DNA & 2 & $10,791.70$ & $6,661.90$ \\
High integrity DNA & 3 & $9,479.40$ & $10,613.80$ \\
High integrity DNA & 4 & $8,428.50$ & $9,964.50$ \\
Rescue sample & 5 & $8,192.40$ & $9,191.60$ \\
Rescue sample & 6 & $10,124.70$ & $9,997.10$ \\
Rescue sample & 7 & $9,608.30$ & $7,981.90$ \\
Rescue sample & 8 & $6,824.40$ & $9,133.50$ \\
Rescue sample & 9 & $8,106.30$ & $3,035.20$ \\
Poor integrity DNA & 10 & $5,521.80$ & $2,075.50$ \\
Poor integrity DNA & 11 & $4,835.40$ & 409.37 \\
Poor integrity DNA & 12 & $3,426.10$ & $1,017.20$ \\
Poor integrity DNA & 13 & $3,676.80$ & $1,526.20$ \\
Poor integrity DNA & 14 & $3,604.90$ & $6,304.10$ \\
Poor integrity DNA & 15 & $1,753.80$ & 134.8 \\
Poor integrity DNA & 16 & $2,597.10$ & $1,595.50$ \\
Poor integrity DNA & 17 & $3,576.10$ & 53.80 \\
& & & \\
\hline
\end{tabular}

Table II. Library yields for libraries prepped with input based on DS1, DS2 and TaqMan RNase P.

\begin{tabular}{|c|c|c|c|}
\hline \multirow[b]{2}{*}{ Sample ID } & \multicolumn{3}{|c|}{ Library yield by input method (pM) } \\
\hline & RNase $\mathrm{P}$ & DS1 & DS2 \\
\hline 1 & 542.4 & 1291.6 & 306.5 \\
\hline 2 & 1970.4 & 1376.8 & 1525 \\
\hline 3 & 4637.7 & 10905.7 & 2155.4 \\
\hline 4 & 704.5 & 1943.3 & 357.7 \\
\hline 5 & 1230.1 & 1816.3 & 439.8 \\
\hline 6 & 1378.1 & 2422.2 & 620.1 \\
\hline 7 & 2881.4 & 4330.8 & 1047.8 \\
\hline 8 & 1625.3 & 4161.4 & 1371.5 \\
\hline 9 & 2475.6 & 5397 & 3246.6 \\
\hline 10 & 52.2 & 1500.9 & 79.8 \\
\hline 11 & 2011.9 & 4558.2 & 2011.4 \\
\hline 12 & 7553.3 & 8535.1 & N/A \\
\hline 13 & 6594.6 & 8391.7 & N/A \\
\hline 14 & 3859.7 & 2578.1 & N/A \\
\hline 15 & 6461.3 & 9272.6 & $\mathrm{~N} / \mathrm{A}$ \\
\hline 16 & 4096.4 & 5847.4 & N/A \\
\hline 17 & 8079.7 & 11336.4 & N/A \\
\hline 18 & 3144.1 & 3582.5 & N/A \\
\hline 19 & 2485.2 & 3836.4 & N/A \\
\hline 20 & 3064.1 & 4629.1 & N/A \\
\hline 21 & 2461.1 & 3446.5 & N/A \\
\hline 22 & 2027.3 & 3462.8 & N/A \\
\hline 23 & 4126.9 & 6945.8 & $\mathrm{~N} / \mathrm{A}$ \\
\hline 24 & 3235.3 & 5278.9 & N/A \\
\hline 25 & 3190.4 & 6423.3 & N/A \\
\hline 26 & 3180.9 & 2834 & N/A \\
\hline 27 & 2500.8 & 4047.5 & N/A \\
\hline 28 & 11203.5 & 5380.5 & N/A \\
\hline 29 & 5465.8 & 7226.6 & $\mathrm{~N} / \mathrm{A}$ \\
\hline 30 & 4961.2 & 3737.4 & N/A \\
\hline 31 & 5029.3 & 6090.5 & N/A \\
\hline 32 & 2502.7 & 3450.3 & N/A \\
\hline 33 & 2913.3 & 4478 & $\mathrm{~N} / \mathrm{A}$ \\
\hline 34 & 3742.7 & 6940.8 & N/A \\
\hline 35 & 3309.1 & 5378.1 & N/A \\
\hline 36 & 2818.1 & 4009.1 & N/A \\
\hline
\end{tabular}

N/A, not available.

SYBR DS2. On average the DNA concentration measured by QC2 was 1.18 times greater than DS2.

Frozen DNA evaluation with the in-house duplex SYBRGreen qPCR compared to FFPE DNA evaluation with in-house duplex SYBR-Green qPCR. On average, the DNA extracted from 26 frozen lung cancer tissue provided higher yield compared to DNA derived from FFPE tissue and lower Dscores. The average DS1 concentration for fresh-frozen DNA 

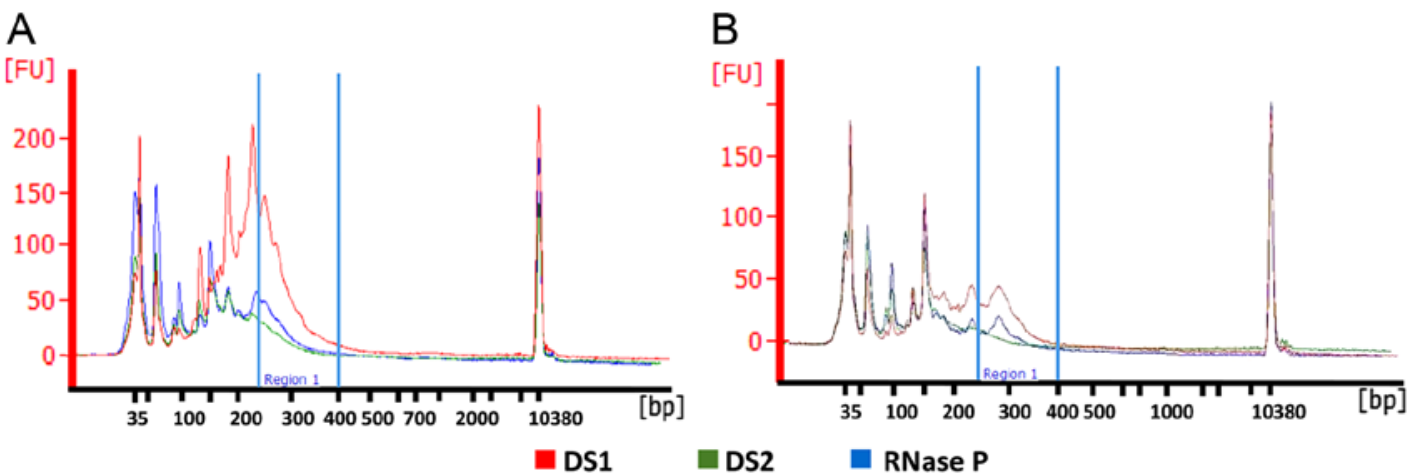

Figure 5. Comparison of NGS libraries yield generated with input based on DS1, DS2 and RNase P quantitation. Targeted NGS panel library electropherograms generated using $10 \mathrm{ng}$ inputs based on quantitation by SYBR-Green DS1, SYBR-Green DS2 and TaqMan RNase P. (A) Overlaid electropherograms for the libraries constructed with $10 \mathrm{ng}$ inputs based on, SYBR-Green DS1 (red), SYBR-Green DS2 (green), and TaqMan RNase P (blue) from a sample with a Dscore of 1.43. (B) Overlaid electropherograms for the libraries constructed with $10 \mathrm{ng}$ inputs based on TaqMan RNase P (blue), SYBR-Green DS1 (red), and SYBR-Green DS2 (green) from a sample with a Dscore of 5.16.

Table III. Sequencing result mutation report for clinical sample P12.

\begin{tabular}{llllcrrr}
\hline $\begin{array}{l}\text { Gene } \\
\text { symbol }\end{array}$ & \multicolumn{1}{c}{ Cosmic ID } & $\begin{array}{c}\text { CDS } \\
\text { mutation }\end{array}$ & $\begin{array}{c}\text { Amino acid } \\
\text { mutation }\end{array}$ & Chromosome & $\begin{array}{c}\text { Mutation } \\
\text { reads }\end{array}$ & $\begin{array}{c}\text { Wild-type } \\
\text { reads }\end{array}$ & $\begin{array}{c}\text { Mutation } \\
\text { Allele Freq. }\end{array}$ \\
\hline MAP2K1 & COSM1562837 & c.158T>G & p.F53V & chr15 & 213 & 1301 & 14.0687 \\
DNMT3A & COSM231571 & c.1502A $>$ G & p.N501S & chr2 & 1349 & 2147 & 38.587 \\
HRAS & COSM249860 & c.81T>C & p.H27H & chr11 & 2811 & 2068 & 57.6143 \\
EGFR & COSM1451600 & c.2361G $>$ A & p.Q787Q & chr7 & 4208 & 6 & 99.8576 \\
\hline
\end{tabular}

CDS, coding DNA sequence; Freq, frequency.

was $21.59 \mathrm{ng} / \mu \mathrm{l}$, while the average DS1 concentration of FFPE DNA was $1.15 \mathrm{ng} / \mu \mathrm{l}$. The average Dscore for frozen DNA was 1.07, while the FFPE DNA averaged 2.34 (data not shown).

Comparison of FFPE DNA extraction methods using in-house duplex SYBR-Green $q P C R$. FFPE DNA extracted using a column based purification method showed similar yield and Dscores to the FFPE DNA extracted using the method without purification when quantified with the in-house SYBR-Green qPCR assay. The FFPE DNA using a column based purification had an average DS1 concentration of $4.94 \mathrm{ng} / \mu \mathrm{l}$ and an average Dscore of 1.48 . When the same tissue samples were extracted using the method without purification the average DS1 concentration was $4.58 \mathrm{ng} / \mu 1$ and the average Dscore was 1.18 (data not shown).

Library preparation comparison of duplex TaqMan to TaqMan RNase P. Samples with Dscores between 0.95 and 1.50 , categorized as 'High integrity DNA' samples, produced similar library yields of targeted fragments of NGS libraries, both for RNase P (10,009.90 pM) and QC2 (9,922.60 pM) input methods. Three of the 4 'high integrity DNA' samples showed greater yields for libraries using input based on QC2 compared to library yields using input based on RNase $\mathrm{P}$ (Table I).

The NGS libraries in three out of five samples categorized as 'Rescue samples', were more productive than those prepared based on QC2. The mean library yields for 'Rescue samples' were 8,571.22 and 7,867.86 pM for input based on RNase $\mathrm{P}$ and QC2, respectively (Table I).

The sample categorized as 'Poor integrity DNA' samples, with high Dscores (>4) or no Cq value for QC2 amplicon, had greater yields with RNase $\mathrm{P}$ inputs compared to libraries made using QC2 input values. The mean library yields for 'Poor integrity DNA' samples were 3,624.00 and 1,639.56 pM input based on RNase P and QC2, respectively. Only 1 of the 8 'Poor integrity DNA' samples showed greater yields for libraries based on QC2 compared to 7 of 8 showing greater yields for libraries based on RNase P (Table I).

Comparison of library preparation of SYBR-Green DS1 and DS2 to TaqMan RNase P. NGS libraries made based on DS2 quantification, had the lowest yields, while DS1 based libraries showed similar or greater yields for most samples when compared to libraries using quantitation by RNase P (Fig. 5 and Table II). The average library yields for the initial 11 samples based on inputs using the DS1 quantitation values was 3,609.47 pM. When the same samples were used to prepare NGS libraries based on the quantitation values from DS2 and RNase P, the average yields were 1,773.60 and 1,196.51 pM, respectively. Ten of the 11 samples showed greater yields for DS1 compared to DS2 and RNase P (Table II).

The average library yields for the additional 25 samples used to prepare NGS libraries based on DS1 and RNase P were 5,485.58 and 4,320.27 pM, respectively (Table II). Twenty-two of the 25 samples showed higher yields when 

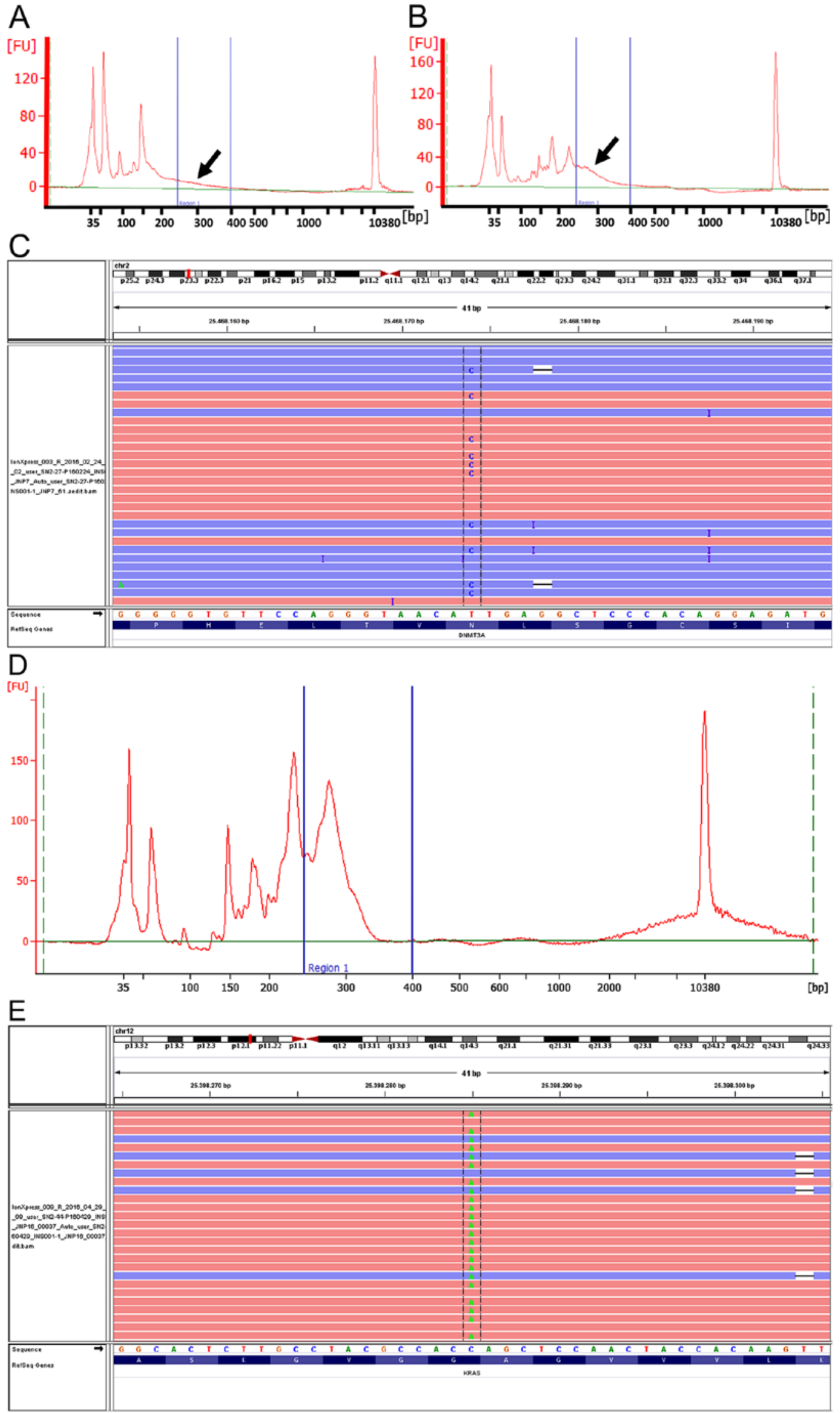

Figure 6. Library yield of targeted NGS library prepared by using DS1-based DNA quantification in clinical samples. (A) Electropherogram of the libraries performed with a $10 \mathrm{ng}$ of input DNA based on the DS1 concentration using the procedure described in Materials and methods. (B) Electropherogram of the libraries performed using $5 \mu \mathrm{l}$ of stock DNA. (C) Integrative Genomics Viewer (IGV) tracks of the non-synonymous DNMT3A p.N501S mutation found in clinical sample P12. (D) Electropherogram of the library generated from a 10 ng DNA input based on the DS1 concentration using the procedure described in the methods section for a sample with a Dscore of 1.37. (E) IGV tracks of the non-synonymous KRAS p.G12C mutation found in sample P37. 
prepared with DS1 inputs compared to the corresponding library prepared with RNase P based inputs. Of the total 36 libraries made with quantitation based on DS1 and RNase P, DS1 provided the greatest yields for 32 out of the 36 samples tested $(89 \%)$, while RNase $\mathrm{P}$ quantitation input method provided the greatest yields in 4 out of the 36 samples $(11 \%)$. Examples of the targeted NGS library preparation results from high and medium-bad quality of DNA are shown in Fig. 5.

Clinical significance. Using the dual-primer pair SYBR-Green qPCR assay to quantify and assess quality of FFPE DNA we were able to obtain sequencing data and mutation results for a clinical sample taken from a gastric mass indicated as upper GI/pancreaticobiliary adenocarcinoma (clinical P12) that would not have been possible using a single primer quantitation method. The concentration of sample P12 quantified by DS1 was 7.20 and $0.03 \mathrm{ng} / \mu \mathrm{l}$ by DS2. The ratio of DS1 to DS2 generated a Dscore of 226.67. NGS libraries were prepared by using $10 \mathrm{ng}$ input based on DS1 concentration and by using the maximum input possible ( $5 \mu 1$ of stock DNA), due to the low concentration of DS2 and the degradation indicated by the high Dscore. The library prep performed based on the DS1 concentration quantified at $444.09 \mathrm{pM}$ for the target library products (Fig. 6A). The library prep performed with the maximum input of $5 \mu \mathrm{l}$ of stock DNA quantified was $1,174.9 \mathrm{pM}$ for the library target regions (Fig. 6B). Sequencing of the maximum DNA input library revealed four mutations including two non-synonymous mutations MAP2K1 p.F53V and DNMT3A p.N501S (Fig. 6C and Table III). Fig. 6D and E show library yield and non-synonymous mutation KRAS p.G12C found in sample P37.

\section{Discussion}

We initially considered six DNA measurement methods for targeted NGS. In addition to four methods tested in the present study, UV spectrophotometer (i.e. NanoDrop) and a chip-based capillary electrophoresis (i.e. Agilent Bioanalyzer) were initially considered, but not selected for this testing. UV spectrophotometer is the easiest and cheapest method among all and would be ideal if it provided accurate results. However, in our previous testing, the measured DNA amount was significantly overestimated causing a high NGS library failure rate or lower library amplification rate due to a small amount of DNA input (data not shown). This is not surprising because it is known that UV spectrophotometers measure all components that absorb within the designated wavelength ranges $(20,30)$. Thus, it is not recommended to use a UV spectrophotometer for assessing DNA quantity for NGS analyses especially for challenging clinical samples. The Bioanalyzer may be a gold standard for RNA quantity and quality measurement for transcriptome sequencing (RNAseq) or gene expression microarray (31). The RNA integrity number (RIN) provided by Bioanalyzer is regarded as a standard for quality assessment of RNA (31). We also use this method for checking the NGS library quality and quantity as it provides not only quantity, but also size and quality pattern $(6,7)$. As targeted NGS library preparation using amplification involves a ligation step of adaptors or barcodes to the amplified DNA, it is critical to see the correct size and the pattern of ligated products. For this purpose, the Bioanalyzer is powerful and would be one of the best methods for NGS library quality check $(6,7)$. However, we did not select this method for DNA quality and quantity assessment for targeted NGS. First, it requires multiple experimental steps and takes relatively longer experimental time. Second, its cost is also higher than other methods. Third, the throughput with a regular Bioanalyzer is limited to 11 samples excluding the ladder control per run. As our goal for developing DNA quality and quantity assessment method is to make a highly accurate and easy-to-use assay with a reasonably low cost, we excluded UV spectrophotometer and Bioanalyzer and tested four remaining methods against TaqMan RNase $\mathrm{P}$ as a control reference.

First, we tested PicoGreen fluorometry using FFPE clinical samples and compared the results with TaqMan RNase $\mathrm{P}$ assay. We tested FFPE DNA with around 0.5-35 $\mathrm{ng} / \mu \mathrm{l}$ concentration and found a strong correlation between the two methods $\left(\mathrm{R}^{2}=0.37\right.$; Fig. 2A). Then we further tested 1:5 diluted DNAs in order to check whether a small amount of DNA from clinical samples can be correctly repeated and found worse correlation $\left(\mathrm{R}^{2}=0.28\right.$; Fig. $\left.2 \mathrm{~B}\right)$.

Another test using a Qubit ${ }^{\circledR}$ fluorometry using a similar approach with PicoGreen method showed a generally lower DNA concentration compared to those from RNase P TaqMan (Fig. 3). This is somewhat surprising as TaqMan detects only 'amplifiable' or 'functionally intact' DNAs while Qubit fluorometer method detects bulk dsDNA (Fig. 3).

Next, we tested dual-probe qPCR system using frozen and FFPE clinical samples. The goal of using two qPCR probes is to measure degradation degree of DNA and provide better and more accurate quantitation depending on the degradation status. We thoroughly searched and investigated the TCGA copy number data to identify the regions and genes showing no or least amplified or deleted in various cancers and identified three loci, 22q12.3, 14q24.1 and 15q24.3. We designed probes targeting two amplicons with different size for both TaqMan and SYBR-Green chemistries. Clinical FFPE samples were tested by TaqMan (Fig. 4A and B) and SYBR-Green (Fig. 4C and D) methods. Quantification based on small amplicons in both platforms (Fig. 4A and C) showed strong correlations with RNase P data. Clinical frozen samples were tested with SYBR-Green method. The frozen DNA yield compared to the yield of the FFPE DNA was higher. The Dscore of the frozen DNA samples indicated high quality intact DNA as expected from the frozen sample type. We also compared a small set of samples from two different FFPE DNA extraction methods with the SYBR-Green assay. The extraction method did not affect the average DS1 or Dscore results. This suggests the SYBR-Green method is suitable assessment of DNA from various extraction methods.

We then made targeted NGS libraries based on TaqMan RNase P and TaqMan QC2 quantification methods with the clinical FFPE DNA samples. TaqMan QC2 quantification did not generate libraries with greater yields than libraries made using TaqMan RNase P quantification (Table I). This suggests that QC2 is not a suitable indicator of DNA quantitation for library preparation.

We then made targeted NGS libraries based on three quantification methods, TaqMan RNase P, SYBR-Green DS1 
(small amplicon), and SYBR-Green DS2 (large amplicon) (Fig. 5). One high quality DNA (Fig. 5A) and a relatively degraded and low quality of DNA (Fig. 5B) were used for targeted NGS library preparation. There was a big difference between SYBR-Green DS1-based quantification and others for the library ligation efficiency in a high quality of DNA (Fig. 5A) while a relatively smaller but still better ligation efficiency was found in SYBR-Green DS1-based quantification in a low quality sample (Fig. 5B). This suggests that our designed SYBR-Green DS1-based DNA quantification provides a good indication for a various range of DNA samples in term of their quality. The additional 34 FFPE samples with different Dscores (degradation degrees, D=DS1/DS2) were tested and showed similar results as shown in Table I.

Next, we identified one clinical sample, with a very high DNA degradation, requested for targeted NGS analysis in a clinical laboratory. This sample (P12) had a Dscore of 226.67 suggesting a very high degradation and poor quality of DNA. In order to maximize the ligation efficiency, two different library preparations were processed based on two DNA quantifications. First is the original DS1-based quantification (Fig. 6A) and the other is 3.6-times more DNA input based on the extremely high Dscore on this sample (Fig. 6B). As shown in Fig. 6A, almost nothing was found in the targeted size range in the original quantification without considering Dscore or DNA degradation degree. However, by considering the DNA degradation degree and adding more DNA, a better and improved ligation efficiency was obtained (Fig. 6B). The subsequent sequencing of the increased DNA library preparation was done well, satisfying our standard and QC step (Fig. 6C). This indicates the usefulness of Dscore by calculating DNA quantification in two different sizes of amplicons. Samples with high Dscore (high degradation) may need to be considered for a different NGS ligation preparation protocol such as higher input DNA or different amplification condition or ligation condition.

In summary, we tested four different DNA quantification methods and compared them with RNase P TaqMan assay to identify the best DNA analysis method for targeted NGS library preparation. We concluded that SYBR-Green-based qPCR assay provides accurate results in a cost effective way for DNA quantification for NGS analyses. Our dual probe qPCR assay also provides a DNA degradation ratio so that NGS library preparation can be optimized based on the degradation status.

\section{Acknowledgements}

The present study was supported in part by funds from the Thoracic Oncology Laboratory at University of California San Francisco (UCSF) and CureSeq Inc.

\section{References}

1. Bennett ST, Barnes C, Cox A, Davies L and Brown C: Toward the 1,000 dollars human genome. Pharmacogenomics 6: 373-382, 2005.

2. Hillier LW, Marth GT, Quinlan AR, Dooling D, Fewell G, Barnett D, Fox P, Glasscock JI, Hickenbotham M, Huang W, et al: Whole-genome sequencing and variant discovery in C. elegans. Nat Methods 5: 183-188, 2008
3. Parsons DW, Jones S, Zhang X, Lin JC, Leary RJ, Angenendt P, Mankoo P, Carter H, Siu IM, Gallia GL, et al: An integrated genomic analysis of human glioblastoma multiforme. Science 321: 1807-1812, 2008.

4. Shah SP, Morin RD, Khattra J, Prentice L, Pugh T, Burleigh A, Delaney A, Gelmon K, Guliany R, Senz J, et al: Mutational evolution in a lobular breast tumour profiled at single nucleotide resolution. Nature 461: 809-813, 2009.

5. Fang LT, Lee S, Choi H, Kim HK, Jew G, Kang HC, Chen L, Jablons D and Kim IJ: Comprehensive genomic analyses of a metastatic colon cancer to the lung by whole exome sequencing and gene expression analysis. Int J Oncol 44: 211-221, 2014.

6. Kang HC, Kim HK, Lee S, Mendez P, Kim JW, Woodard G, Yoon JH, Jen KY, Fang LT, Jones K, et al: Whole exome and targeted deep sequencing identify genome-wide allelic loss and frequent SETDB1 mutations in malignant pleural mesotheliomas. Oncotarget 7: 8321-8331, 2016.

7. Mendez P, Dang J, Kim JW, Lee S, Yoon JH, Kim T, Sailey CJ, Jablons DM and Kim IJ: Comprehensive evaluation and validation of targeted next-generation sequencing performance in two clinical laboratories. Int J Oncol 49: 235-242, 2016.

8. Hadd AG, Houghton J, Choudhary A, Sah S, Chen L, Marko AC, Sanford T, Buddavarapu K, Krosting J, Garmire L, et al: Targeted, high-depth, next-generation sequencing of cancer genes in formalin-fixed, paraffin-embedded and fine-needle aspiration tumor specimens. J Mol Diagn 15: 234-247, 2013.

9. Han SW, Kim HP, Shin JY, Jeong EG, Lee WC, Lee KH, Won JK, Kim TY, Oh DY, Im SA, et al: Targeted sequencing of cancer-related genes in colorectal cancer using next-generation sequencing. PLoS One 8: e64271, 2013.

10. Cancer Genome Atlas Research Network: Comprehensive genomic characterization defines human glioblastoma genes and core pathways. Nature 455: 1061-1068, 2008.

11. Kandoth C, McLellan MD, Vandin F, Ye K, Niu B, Lu C, Xie M, Zhang Q, McMichael JF, Wyczalkowski MA, et al: Mutational landscape and significance across 12 major cancer types. Nature 502: 333-339, 2013.

12. Brennan CW, Verhaak RG, McKenna A, Campos B, Noushmehr H, Salama SR, Zheng S, Chakravarty D, Sanborn JZ, Berman SH, et al; TCGA Research Network: The somatic genomic landscape of glioblastoma. Cell 155: 462-477, 2013.

13. Bass AJ, Thorsson V, Shmulevich I, Reynolds SM, Miller M, Bernard B, Hinoue T, Laird PW, Curtis C, Shen H, et al; Cancer Genome Atlas Research Network: Comprehensive molecular characterization of gastric adenocarcinoma. Nature 513: 202-209, 2014.

14. Imielinski M, Berger AH, Hammerman PS, Hernandez B, Pugh TJ, Hodis E, Cho J, Suh J, Capelletti M, Sivachenko A, et al: Mapping the hallmarks of lung adenocarcinoma with massively parallel sequencing. Cell 150: 1107-1120, 2012

15. Ng SB, Turner EH, Robertson PD, Flygare SD, Bigham AW, Lee C, Shaffer T, Wong M, Bhattacharjee A, Eichler EE, et al: Targeted capture and massively parallel sequencing of 12 human exomes. Nature 461: 272-276, 2009.

16. Choi M, Scholl UI, Ji W, Liu T, Tikhonova IR, Zumbo P, Nayir A, Bakkaloğlu A, Ozen S, Sanjad S, et al: Genetic diagnosis by whole exome capture and massively parallel DNA sequencing. Proc Natl Acad Sci USA 106: 19096-19101, 2009.

17. Ng SB, Buckingham KJ, Lee C, Bigham AW, Tabor HK, Dent KM, Huff CD, Shannon PT, Jabs EW, Nickerson DA, et al: Exome sequencing identifies the cause of a mendelian disorder. Nat Genet 42: 30-35, 2010.

18. Gawad C, Koh W and Quake SR: Single-cell genome sequencing: Current state of the science. Nat Rev Genet 17: 175-188, 2016.

19. de Bourcy CF, De Vlaminck I, Kanbar JN, Wang J, Gawad C and Quake SR: A quantitative comparison of single-cell whole genome amplification methods. PLoS One 9: e105585, 2014.

20. Nielsen K, Mogensen HS, Hedman J, Niederstätter H, Parson W and Morling N: Comparison of five DNA quantification methods. Forensic Sci Int Genet 2: 226-230, 2008.

21. Navarro E, Serrano-Heras G, Castano MJ and Solera J: Real-time PCR detection chemistry. Clin Chim Acta 439: 231-250, 2015.

22. Singer VL, Jones LJ, Yue ST and Haugland RP: Characterization of PicoGreen reagent and development of a fluorescence-based solution assay for double-stranded DNA quantitation. Anal Biochem 249: 228-238, 1997.

23. Robin JD, Ludlow AT, LaRanger R, Wright WE and Shay JW: Comparison of DNA quantification methods for next generation sequencing. Sci Rep 6: 24067, 2016. 
24. White RA III, Blainey PC, Fan HC and Quake SR: Digital PCR provides sensitive and absolute calibration for high throughput sequencing. BMC Genomics 10: 116, 2009.

25. Panaro NJ, Yuen PK, Sakazume T, Fortina P, Kricka LJ and Wilding P: Evaluation of DNA fragment sizing and quantification by the agilent 2100 bioanalyzer. Clin Chem 46: 1851-1853, 2000.

26. Arya M, Shergill IS, Williamson M, Gommersall L, Arya N and Patel HR: Basic principles of real-time quantitative PCR. Expert Rev Mol Diagn 5: 209-219, 2005.

27. Buh Gasparic M, Tengs T, La Paz JL, Holst-Jensen A, Pla M, Esteve T, Zel J and Gruden K: Comparison of nine different real-time PCR chemistries for qualitative and quantitative applications in GMO detection. Anal Bioanal Chem 396: 2023-2029, 2010 .
28. Vogelstein B, Papadopoulos N, Velculescu VE, Zhou S, Diaz LA Jr and Kinzler KW: Cancer genome landscapes. Science 339: 1546-1558, 2013.

29. Karro JE, Yan Y, Zheng D, Zhang Z, Carriero N, Cayting P, Harrrison P and Gerstein M: Pseudogene.org: A comprehensive database and comparison platform for pseudogene annotation. Nucleic Acids Res 35 (Database): D55-D60, 2007.

30. Bhat S, Curach N, Mostyn T, Bains GS, Griffiths KR and Emslie KR: Comparison of methods for accurate quantification of DNA mass concentration with traceability to the international system of units. Anal Chem 82: 7185-7192, 2010.

31. Kim IJ, Quigley D, To MD, Pham P, Lin K, Jo B, Jen KY, Raz D, $\mathrm{Kim} \mathrm{J}$, Mao JH, et al: Rewiring of human lung cell lineage and mitotic networks in lung adenocarcinomas. Nat Commun 4: 1701,2013 